\title{
3D-DIR for early differential diagnostic and prognostic evaluation of NMO
}

\author{
YANBING WANG ${ }^{1,2}$, HONG YAN ${ }^{2}$, QIXING DING ${ }^{2}$, CUNHUA MAO $^{3}$, \\ YELONG SHEN $^{1}$ and GUANGBIN WANG ${ }^{1}$
}

\author{
${ }^{1}$ Shandong Medical Imaging Research Institute, Shandong University, Jinan, Shandong 250021; ${ }^{2}$ Department of Radiology, \\ Rizhao People's Hospital, Rizhao, Shandong 276826; ${ }^{3}$ The Affiliated Hospital of Shandong University \\ of Traditional Chinese Medicine, Jinan, Shandong 250012, P.R. China
}

Received February 18, 2016; Accepted June 23, 2016

DOI: $10.3892 /$ etm.2016.3474

\begin{abstract}
Neuromyelitis optica (NMO) is an acute or subacute lesion of demyelinating disease involving the optic nerve and spinal cord, and imaging techniques and their effects have been the focus of investigations. The aim of the present study was to examine the value of three-dimensional double inversion recovery (3D-DIR) in the early differential diagnostic and prognostic evaluation of NMO. Forty-eight patients with suspicious NMO were included into the study and underwent a combination of serum NMO-IgG quantitative detection and 3D-DIR examination. Forty cases $(83.3 \%)$ of the suspicious cases were confirmed with NMO. The average time from onset to definite diagnosis was $3.5 \pm 0.6$ days. The brain showed high T2W and fluid-attenuated inversion recovery (FLAIR) signals, involving $5.8 \pm 1.2$ sites on average, distributed in the peripheral lateral ventricle, medulla, cerebral white matter, the third ventricle, peripheral aqueduct of sylvius, pons and diencephalon. The average T2W signal strength was $2.73 \pm 0.12$. The signal intensity of DIR was significantly higher than that of T2W and FLAIR, and the difference was statistically significant. The optic nerve and chiasma showed a high FLAIR signal, with an average signal intensity of $2.13 \pm 0.14$. The spinal cord showed swelling, necrosis and cavity lesion, involving the gray and white matter of the central site, transversely, with an average lesion length of $4.7 \pm 0.6$ centrum. The relative signal intensity of DIR was significantly higher than that of T2W and FLAIR. Following treatment, the signal intensity of the brain, optic nerve, optic chiasma and spinal cord decreased significantly $(\mathrm{P}<0.05)$. In conclusion, 3D-DIR has great application value in the early differential diagnostic and prognostic evaluation of NMO.
\end{abstract}

Correspondence to: Dr Guangbin Wang, Shandong Medical Imaging Research Institute, Shandong University, 324 Jing Wu Road, Jinan, Shandong 250021, P.R. China

E-mail: fmppumnwii396@163.com

Key words: three-dimensional double inversion recovery, neuromyelitis optica, signal intensity

\section{Introduction}

Neuromyelitis optica (NMO) is an acute or subacute lesion of demyelinating disease involving optic nerve and spinal cord (1). Previously, NMO was considered a clinical subtype of multiple sclerosis (MS), although the effects of clinical treatment were unsatisfactory $(1,2)$. The development of imaging technology identified NMO and MS as different with regard to the characteristics of lesions.

By applying two inversion pulses, three-dimensional double inversion recovery (3D-DIR) sequence significantly improved the signal contrast of different tissues and the imaging effects of gray matter lesions (3). In recent years, 3D-DIR has been gradually applied in the diagnosis of epilepsy, MS and other diseases, and the effects have proven to be satisfactory (4-6).

The aim of the present study was to examine the application value of 3D-DIR in the early differential diagnostic and prognostic evaluation of NMO.

\section{Materials and methods}

Patients. In total, 48 patients diagnosed with suspicious NMO at the Rizhao People's Hospital between October 2013 and October 2015 were included into the present study. All 48 patients were confirmed to be first onset and first treatment according to the clinical symptoms and physical signs. Of the 48 patients, there were 10 males and 38 females including 7 children. The patient age range was 8-66 years, with a median age of 43.5 years, and a time of onset ranging from 1 to 3 days with an average of $1.7 \pm 0.5$ days. The patients with central nervous demyelinating lesions such as MS, definite infection history, cerebral tumor, epilepsy, pregnancy, consciousness disturbance and those who refused to participate were excluded.

Approval for the present study was obtained from the ethics committee of the Rizhao People's Hospital. Patients and their families/guardians provided informed consent.

Methods. The patients were given a combined NMO-IgG quantitative detection and 3D-DIR examination as well as standard medical drug therapy. For the 3D-DIR examination, a Siemens MAGNETOM Skyra 3.0T superconducting scanner (Siemens Healthcare GmbH, Erlangen, Germany) 

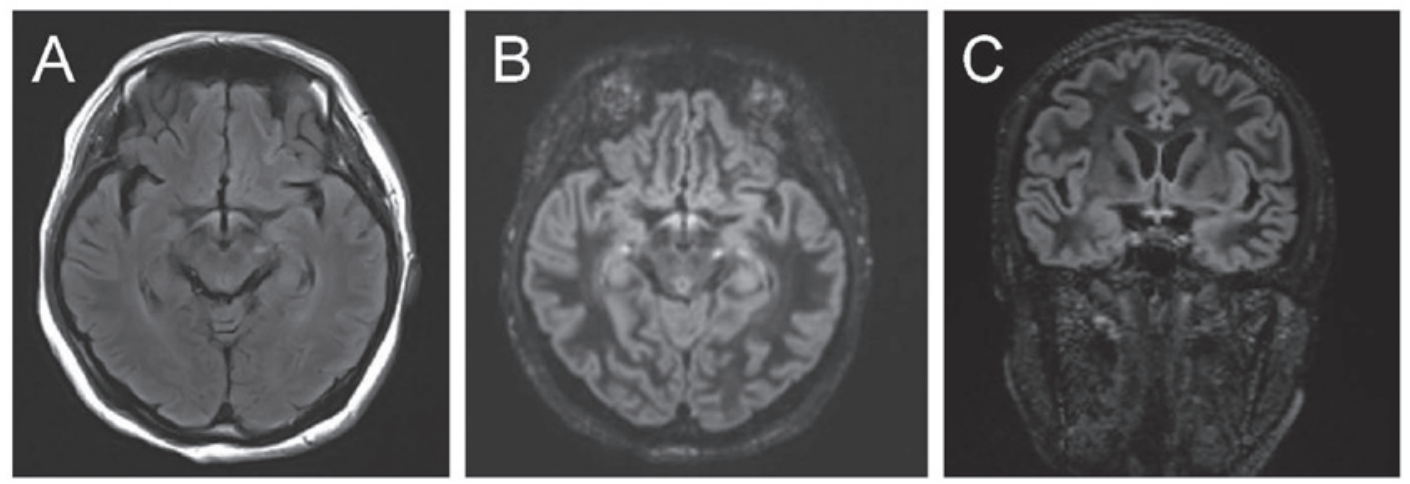

Figure 1. (A) Optic chiasma showed high FLAIR signal with an average signal intensity of $2.13 \pm 0.14$. (B and C) DIR transverse and coronal view shows higher signals with an average signal intensity of $2.37 \pm 0.23$. FLAIR, fluid-attenuated inversion recovery; DIR, double inversion recovery.

and 8-channel head coil were used to perform the routine MR examination. Fast spin echo (TSE) T2WI, fluid-attenuated inversion recovery (FLAIR) sequence and 3D-DIR sequence were examined in turn. Parameters of the 3D-DIR sequence included (TR 7,500 msec, TE $308 \mathrm{msec}$; T1 3,000 msec; matrix 190x192; visual field of FOV 256x256 mm; 128 layers; and thickness of $1.3 \mathrm{~mm}$ ), two reversal pulses, TI1 (time from the first $180^{\circ}$ reverse pulse to $90^{\circ}$ drive pulse) $3,400 \mathrm{msec}$, and parameters of TI2 (time from the second $180^{\circ}$ reverse pulse to $90^{\circ}$ drive pulse) $325 \mathrm{msec}$. Subsequently, T2W TSE sequence (TR 5,000 msec, TE 87 msec; matrix 256x256; FOV 256x256 mm; 35 layers; thickness of $4 \mathrm{~mm}$ ) scanning was performed. According to the anatomical location of the lesions in MRI, the lesions were divided into: endodermal lesions, gray and white matter mixed lesions, subcortical lesions, deep gray matter lesions and white matter lesions.

Observation indexes. The signal characteristics of the 3D-DIR scan of the cerebral and spinal cord lesions of NMO and MS patients were compared, and the signal characteristics of 3D-DIR scan of NMO patients prior and subsequent to surgery were compared.

Statistical methods. SPSS 20.0 statistical software (Chicago, IL, USA) was used for statistical analysis. Measurement data were presented as mean \pm standard deviation. Enumeration data were expressed as a percentage (\%). The group comparison was made using the $\chi^{2}$ test. $\mathrm{P}<0.05$ was considered to be statistically significant.

\section{Results}

Signal characteristics of $3 D-D I R$ scaning of NMO patients. The diagnostic criteria for NMOSD revised by Wingerchuk et al in 2015 (7) indicated: i) at least one core clinical characteristic, ii) positive test for AQP4-IgG using the best available detection method, and iii) exclusion of alternative diagnoses. At least one core clinical characteristic (optic nerve) was neuritis and acute myelitis, with the supporting standard (met at least two requirements) being: spinal cord MRI lesion extended at least three segments, head MRI failed MS diagnostic criteria, and positive serum NMO-IgG. Forty cases (83.3\%) were confirmed with NMO, and the time from onset to diagnosis was $3.5 \pm 0.6$ days on average.
Table I. Brain signal characteristics of NMO patients.

\begin{tabular}{lcccc}
\hline Group & T2W & FLAIR & DIR & $\begin{array}{c}\text { Mean } \\
\text { involved } \\
\text { parts }\end{array}$ \\
\hline NMO patients & $2.73 \pm 0.12$ & $2.56 \pm 0.23$ & $2.89 \pm 0.32$ & $5.8 \pm 1.2$ \\
MS patients & $2.84 \pm 0.15$ & $2.77 \pm 0.25$ & $3.04 \pm 0.22$ & $2.7 \pm 0.6$ \\
t & 4.251 & 4.189 & 4.237 & 5.324 \\
P-value & 0.043 & 0.044 & 0.042 & 0.038 \\
\hline
\end{tabular}

NMO, neuromyelitis optica; FLAIR, fluid-attenuated inversion recovery; DIR, double inversion recovery.

The brain of NMO patients showed low T1W signal, high T2W and FLAIR signals, involving 5.8 \pm 1.2 sites on average, and being distributed in the peripheral lateral ventricle, medulla, cerebral white matter, the third ventricle, peripheral aqueduct of sylvius, pons and diencephalon. The average T2W signal strength was $2.73 \pm 0.12$, average FLAIR signal strength was $2.56 \pm 0.23$ and the average DIR signal strength was $2.89 \pm 0.32$. The signal intensity of T2W, FLAIR and DIR of NMO patients was significantly higher than that of MS patients, with the average sites involved being more than those of MS patients $(\mathrm{P}<0.05)$ (Table I and Fig. 1).

Signal characteristics of $3 D-D I R$ scan of NMO patients prior to and after treatment. Following treatment, the signal intensity of the brain, optic nerve, optic chiasma and spinal cord decreased significantly, and the differences were statistically significant $(\mathrm{P}<0.05)$ (Table II, and Figs. 2 and 3).

\section{Discussion}

NMO is a type of demyelinating disease that occurs in the central nerve system. Its pathological features include failure of the nerve fiber myelin sheath and the presence of multiple small disseminated disease or relatively large lesions infused by one or more lesions, distributed in white matter and infiltrating along the inflammatory cells surrounding veinlet; neurons, axons, and supporting tissues that remain relatively intact, free from wallerian degeneration or secondary bundle 
Table II. Signal characteristics of 3D-DIR scan of NMO patients before and after treatment.

\begin{tabular}{lccccccc}
\hline & T2W & FLAIR & Brain 3D-DIR & $\begin{array}{c}\text { Optic nerve } \\
\text { FLAIR }\end{array}$ & $\begin{array}{c}\text { Optic nerve } \\
\text { 3D-DIR }\end{array}$ & $\begin{array}{c}\text { Spinal cord } \\
\text { 3D-DIR }\end{array}$ & $\begin{array}{c}\text { Spinal cord } \\
\text { FLAIR }\end{array}$ \\
\hline Pre-treatment & $2.73 \pm 0.12$ & $2.56 \pm 0.23$ & $2.84 \pm 0.25$ & $2.13 \pm 0.14$ & $2.37 \pm 0.23$ & $2.17 \pm 0.24$ & $1.98 \pm 0.22$ \\
Post-treatment & $1.35 \pm 0.13$ & $1.42 \pm 0.21$ & $1.63 \pm 0.24$ & $1.77 \pm 0.15$ & $1.98 \pm 0.17$ & $1.83 \pm 0.21$ & $1.63 \pm 0.18$ \\
t & 4.647 & 4.795 & 5.321 & 5.102 & 5.624 & 5.698 & 4.968 \\
P-value & 0.037 & 0.036 & 0.032 & 0.034 & 0.021 & 0.017 & 0.035 \\
\hline
\end{tabular}

3D-DIR, three-dimensional double inversion recovery NMO, neuromyelitis optica; FLAIR, fluid-attenuated inversion recovery.
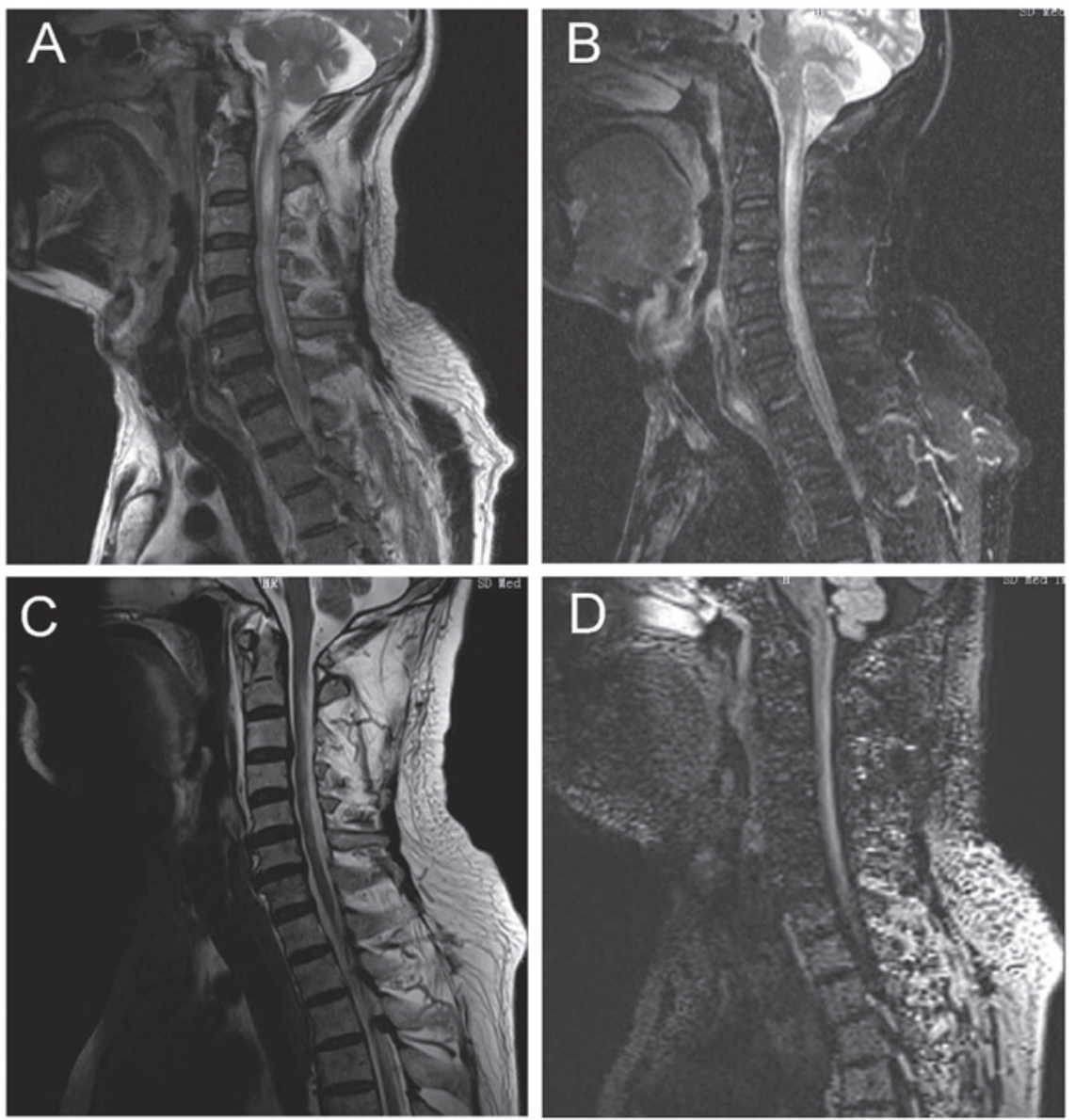

Figure 2. Prior to treatment (A) T2 image; (B) enhanced scanning. After treatment (C) T2 and (D) DIR image shows decreased signal, reduced lesion scope and amyelotrophy. DIR, double inversion recovery.

degeneration (8). The etiology and mechanism of NMO remain unknown, but may be associated with genetic quality and ethnic differences (9). NMO mainly involves the optic nerve, optic chiasma and spinal cord, and lesions appearing in the thoracic and cervical segments, which is different from typical MS. Additionally, its destructive lesions are more obvious and axonal destruction may also be present (10). Although NMO-IgG has $91 \%$ specificity and $73 \%$ sensitivity in the diagnosis of NMO, the NMO-IgG of most MS patients are negative. Nevertheless, in clinic, imaging diagnosis remains the first choice for the early diagnosis of $\mathrm{NMO}(11,12)$.

MRI is the first choice for the imaging diagnosis of NOM, but the spatial and contrast resolution of conventional MR pulse sequences is not efficient and requires improvement in displaying gray matter lesions $(13,14)$. The 3D-DIR pulse sequence uses pulses that may inhibit the cerebrospinal fluid and white matter signals, better display the gray matter of the brain, improve the sensitivity of gray matter lesions, and identify cortical lesions that may not be detected by conventional MR pulse sequence (13-15). In the DIR sequence, selecting two TI values (TI1 and TI2) according to the longitudinal relaxation time (T1) of the tissues to be imaged, and in brain scanning, selecting corresponding TI1 and TI2 values may inhibit cerebrospinal fluid and white matter signals at the same time and better display the gray matter of the brain $(16,17)$. At present, it is rarely used in the study of NMO. 

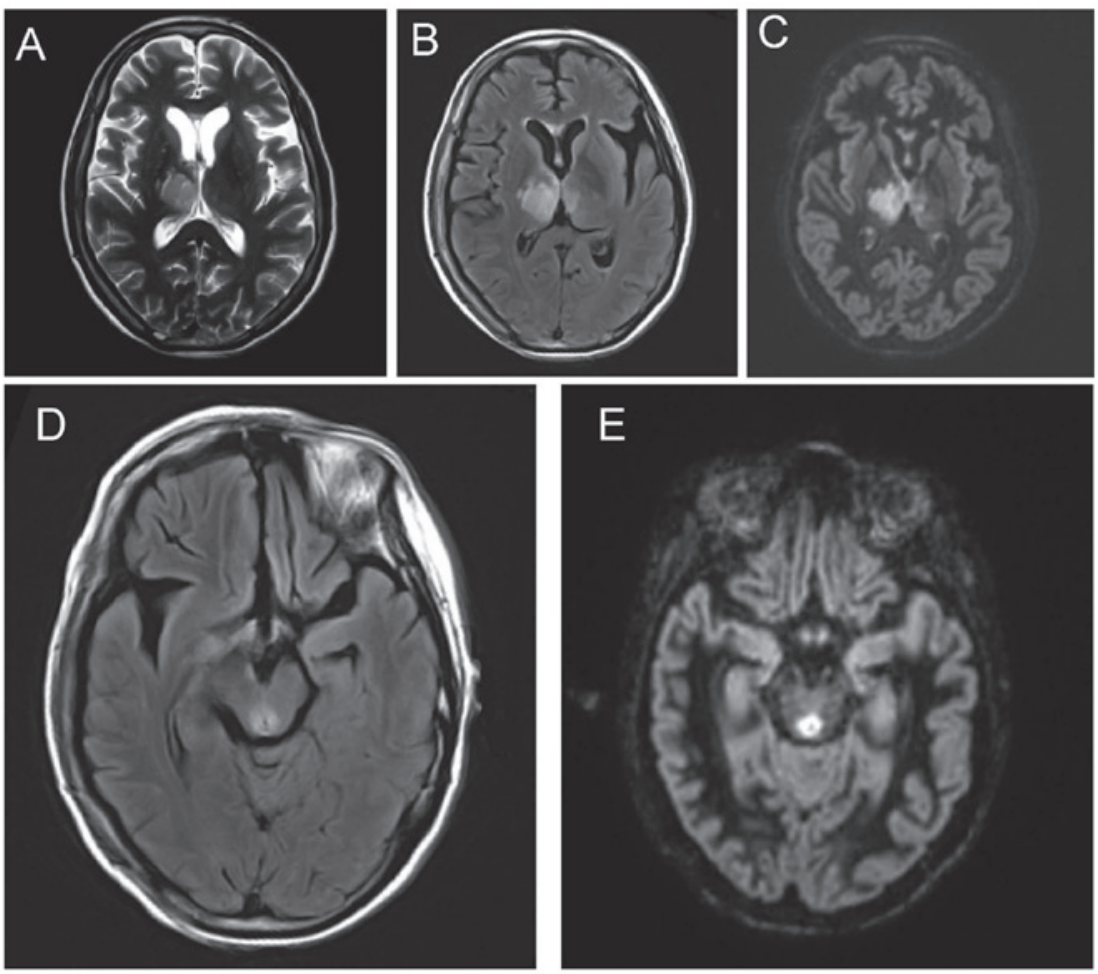

Figure 3. Brain (A) T2WI (B) FLAIR (C) DIR (D) FLAIR and (E) DIR. FLAIR, fluid-attenuated inversion recovery; DIR, double inversion recovery.
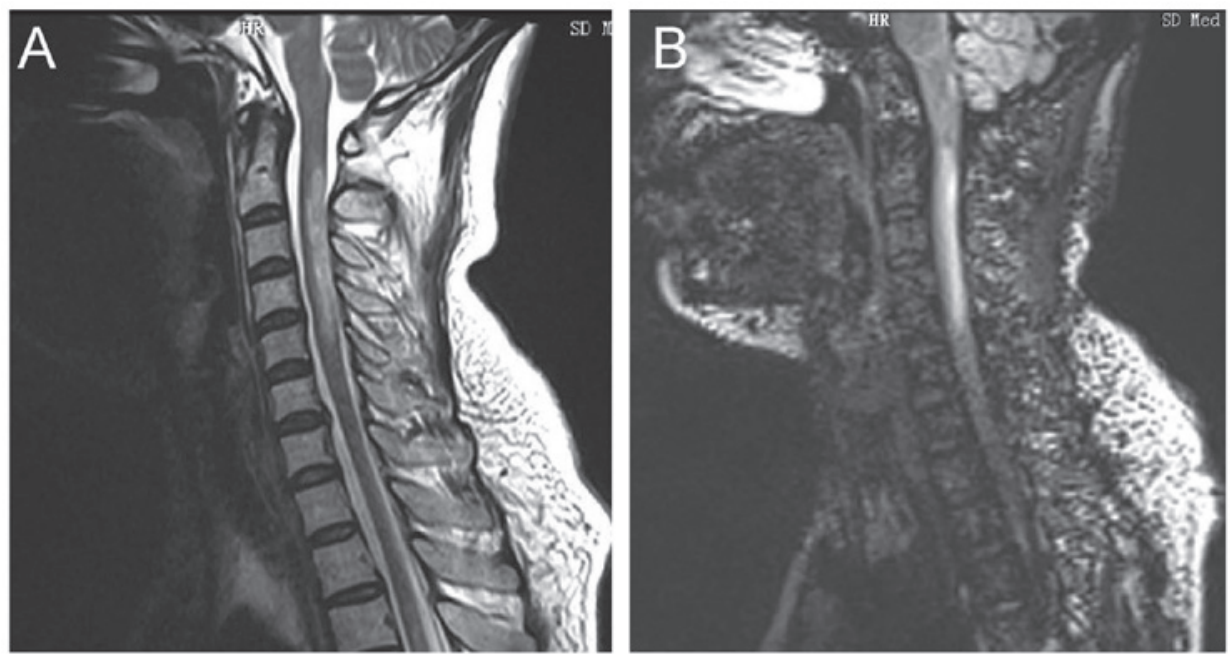

Figure 4. (A) T2WI spinal cord shows swelling, necrosis and cavity lesion, involving the gray and white matter of the central site, transversely, and the average length of the lesion was $4.7 \pm 0.6$ centrum. (B) DIR shows higher signal. Lesions of MS patients were located in the peripheral spinal cord and the average length of the lesion was $0.9 \pm 0.2$ centrum. DIR, double inversion recovery; MS, multiple sclerosis.

In the present study, by comparing the imaging characteristics of NMO and MS, we found that: i) the brain of NMO patients showed a low T1W signal, high T2W and FLAIR signals, involving on average $5.8 \pm 1.2$ sites, distributed in the peripheral lateral ventricle, medulla, cerebral white matter, the third ventricle, peripheral aqueduct of sylvius, pons and diencephalon. AQP4 was similarly highly expressed around the aqueductus Sylvii, ventriculus quartus cerebri and central canal (18). The enhancement of Gd-DTPA was varied, periventricular, pia mater enhancement, corpus callosum linear, and zonal enhancement exhibited specificity (19). The ii) optic nerve and chiasma showed a high FLAIR signal. In the acute period, the spinal cord showed swelling, necrosis and cavity lesions, lesions were enhanced after enhanced scanning, involving primarily gray matters and partial white matters of the central part, transversely, with the average length of the lesion being $4.7 \pm 0.6$ centrum (Fig. 4), mostly located in neck and thoracic cord, and the lesions of cervical segment can extend up to the lower part of the medulla, and during recovery, the spinal cord in lesion site was able to shrink $(19,20)$. The results of the present study show that the signal intensity of T2W, FLAIR and DIR of NMO patients were significantly higher than those of the MS patients, the average of sites involved were significantly more than those of 
the MS patients. In addition, after the follow-up of the present study, it was found that the signal intensity of head, optic nerve, optic chiasma and spinal cord following treatment were significantly lower than those prior to treatment.

In summary, 3D-DIR was not only of great reference value for the early differential diagnosis of NMO, but also valuable for the evaluation of prognosis of patients.

\section{References}

1. Juryńczyk M, Craner M and Palace J: Overlapping CNS inflammatory diseases: differentiating features of NMO and MS. J Neurol Neurosurg Psychiatry 86: 20-25, 2015.

2. Mealy MA, Wingerchuk DM, Greenberg BM and Levy M: Epidemiology of neuromyelitis optica in the United States: a multicenter analysis. Arch Neurol 69: 1176-1180, 2012.

3. Simon B, Schmidt S, Lukas C, Gieseke J, Träber F, Knol DL, Willinek WA, Geurts JJ, Schild HH, Barkhof F, et al: Improved in vivo detection of cortical lesions in multiple sclerosis using double inversion recovery MR imaging at 3 Tesla. Eur Radiol 20: $1675-1683,2010$.

4. Riederer I, Karampinos DC, Settles M, Preibisch C, Bauer JS, Kleine JF, Mühlau M and Zimmer C: Double inversion recovery sequence of the cervical spinal cord in multiple sclerosis and related inflammatory diseases. AJNR Am J Neuroradiol 36: 219-225, 2015.

5. Morimoto E, Kanagaki M, Okada T, Yamamoto A, Mori N, Matsumoto R, Ikeda A, Mikuni N, Kunieda T, Paul D, et al: Anterior temporal lobe white matter abnormal signal (ATLAS) as an indicator of seizure focus laterality in temporal lobe epilepsy: comparison of double inversion recovery, FLAIR and T2W MR imaging. Eur Radiol 23: 3-11, 2013.

6. Vural G, Keklikoğlu HD, Temel Ş, Deniz O and Ercan K: Comparison of double inversion recovery and conventional magnetic resonance brain imaging in patients with multiple sclerosis and relations with disease disability. Neuroradiol J 26 : 133-142, 2013

7. Wingerchuk DM, Banwell B, Bennett JL, Cabre P, Carroll W, Chitnis T, de Seze J, Fujihara K, Greenberg B, Jacob A, et al; International Panel for NMO Diagnosis: International consensus diagnostic criteria for neuromyelitis optica spectrum disorders. Neurology 85: 177-189, 2015.

8. Khanna S, Sharma A, Huecker J, Gordon M, Naismith RT and Van Stavern GP: Magnetic resonance imaging of optic neuritis in patients with neuromyelitis optica versus multiple sclerosis. J Neuroophthalmol 32: 216-220, 2012.
9. Tackley G, Kuker W and Palace J: Tackle yG, Kuker W and Palace J: Magnetic resonance imaging in neuromyelitis optica. Mult Scler 20: 1153-1164, 2014

10. Schneider E, Zimmermann H, Oberwahrenbrock T, Kaufhold F, Kadas EM, Petzold A, Bilger F, Borisow N, Jarius S, Wildemann B, et al: Optical coherence tomography reveals distinct patterns of retinal damage in neuromyelitis optica and multiple sclerosis. PLoS One 8: e66151, 2013.

11. Sinnecker T, Dörr J, Pfueller CF, Harms L, Ruprecht K, Jarius S, Brück W, Niendorf T, Wuerfel J and Paul F: Distinct lesion morphology at 7-T MRI differentiates neuromyelitis optica from multiple sclerosis. Neurology 79: 708-714, 2012.

12. Iyer A, Elsone L, Appleton R and Jacob A: A review of the current literature and a guide to the early diagnosis of autoimmune disorders associated with neuromyelitis optica. Autoimmunity 47: 154-161, 2014

13. Kolber P, Montag S, Fleischer V, Luessi F, Wilting J, Gawehn J, Gröger A and Zipp F: Identification of cortical lesions using DIR and FLAIR in early stages of multiple sclerosis. J Neurol 262: 1473-1482, 2015

14. Coebergh JA, Roosendaal SD, Polman CH, Geurts JJ and van Woerkom TC: Acute severe memory impairment as a presenting symptom of multiple sclerosis: a clinical case study with 3D double inversion recovery MR imaging. Mult Scler 16: 1521-1524, 2010

15. Calabrese M and De Stefano N: Cortical lesion counts by double inversion recovery should be part of the MRI monitoring process for all MS patients: yes. Mult Scler 20: 537-538, 2014.

16. Li Q, Zhang Q, Sun H, Zhang Y and Bai R: Double inversion recovery magnetic resonance imaging at $3 \mathrm{~T}$ : diagnostic value in hippocampal sclerosis. J Comput Assist Tomogr 35: 290-293, 2011.

17. Seewann A, Kooi EJ, Roosendaal SD, Pouwels PJ, Wattjes MP, van der Valk P, Barkhof F, Polman CH and Geurts JJ: Postmortem verification of MS cortical lesion detection with 3D DIR. Neurology 78: 302-308, 2012.

18. Pittock SJ, Weinshenker BG, Lucchinetti CF, Wingerchuk DM, Corboy JR and Lennon VA: Neuromyelitis optica brain lesions localized at sites of high aquaporin 4 expression. Arch Neurol 63: 964-968, 2006.

19. Ito S, Mori M, Makino T, Hayakawa S and Kuwabara S: 'Cloud-like enhancement' is a magnetic resonance imaging abnormality specific to neuromyelitis optica. Ann Neurol 66: 425-428, 2009 .

20. Hodel J, Outteryck O, Bocher AL, Zéphir H, Lambert O, Benadjaoud MA, Chechin D, Pruvo JP, Vermersch P and Leclerc X: Comparison of 3D double inversion recovery and 2D STIR FLAIR MR sequences for the imaging of optic neuritis: pilot study. Eur Radiol 24: 3069-3075, 2014. 